\title{
FANTASTIC PLASTIC MAKES THE QUANTUM LEAP
}

॥ Thilo Stöferle and Rainer F. Mahrt - IBM Research - Zurich, Switzerland - DOI: 10.1051/epn/2014203

A Bose-Einstein condensate is an intriguing state of matter where extensive collective coherence leads to macroscopic quantum phenomena.

It has now been demonstrated using a thin plastic film at room temperature. This provides a new, simple route to experimentally study many-body quantum physics and opens the door for device applications. 
4 Previous page: a polymer layer (yellow) is sandwiched between two Bragg mirrors (red and blue oxide layers). When excited with an ultraviolet laser from below, polaritons are created (green). The laser-like light emitted to the top is a signature of the Bose-Einstein condensate.

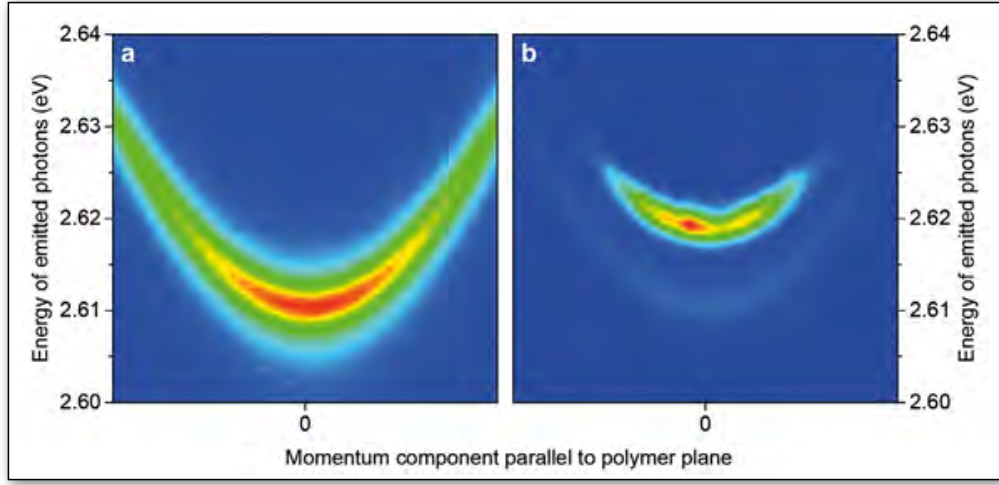

$\triangle$ FIG. 1:

Dispersion relation of the polymer film within the microcavity, where the false colors represent the emitted intensity.

(a) Below the critical polariton density, a parabola with Boltzmann-like intensity distribution in energy and momentum

is observed.

(b) Above the condensation threshold, the polariton BEC shows

up as a separate, narrow peak in the center. The intensity is $\mathbf{1 0 0}$ times higher which requires a corresponding adaptation of the false-color scale.

the the 1920s Satyendranath Bose and Albert Einstein predicted the existence of a new state of matter near the absolute zero temperature. At this low temperature, particles do not behave like point-like billiard balls anymore but acquire significant spatial extension as their de Broglie wavelength increases with decreasing temperature. At sufficiently high density, the wavefunctions of particles whose behaviour in the ensemble follows the so-called Bose-Einstein statistics, start to overlap and accumulate in the lowest energy state. Thereby they lose their individual character and instead form a macroscopic "super-particle", the Bose-Einstein condensate (BEC).

\section{Looking into nature's quantum kitchen}

While it was soon realized that there is an intricate relationship with the phenomena of superfluidity in liquid helium and superconductivity, it took physicists 70 years to create such a state of matter in the laboratory. In 1995, Eric A. Cornell and Carl E. Wieman from JILA in Boulder [1], and Wolfgang Ketterle from MIT in Boston [2] achieved Bose-Einstein condensation in dilute alkali gases at temperatures below a few micro Kelvin, for which they were later awarded the Nobel prize. This breakthrough paved the way for an ongoing, long series of fundamental studies of genuine quantum effects and phases, exploiting the unprecedented possibilities to control, manipulate and investigate this special quantum many-body state. A truly fascinating and appealing aspect of all these experiments is that one can literally really see quantum mechanics "at work".

Shortly after the first BEC, Atac Imamoğlu and co-workers predicted that similar phenomena should be observable also in solid state systems [3]. Here the condensing bosons would be quasi-particles (so-called polaritons) created by strong coupling between bound electron-hole pairs (excitons) and photons in an optical microcavity (see BOX). In contrast to alkali atoms, their number is not conserved as they can readily be created by pumping with a laser and destroyed by recombination of electron-hole pairs or photons leaking out of the cavity. Therefore it is different from Bose and Einstein's original model because the driven and dissipative nature of polaritons intrinsically prohibits thermal equilibrium with the environment. A compelling advantage, however, is that the effective mass of these polaritons is on the order of $10^{10}$ times less than the mass of alkali atoms, which boosts the critical temperature for BEC above room temperature. Furthermore, photons leaking out from the cavity transport all the necessary information about the polariton state to the outside world where they can easily be measured with a spectrograph or imaged by a camera.

\section{From dillute gases at a few micro Kelvin to semiconductors crystals at room temperature}

In 2006, the groups of Benoît Deveaud-Plédran in Lausanne and Le Si Dang in Grenoble jointly demonstrated the first polariton BEC in CdTe quantum wells at $-268^{\circ} \mathrm{C}$ [4]. In the following years, other groups reported polariton $\mathrm{BECs}$ at room temperature, using $\mathrm{GaN}$ or $\mathrm{ZnO}$ structures. The delicate and defect-free growth of these semiconductor crystals and quantum wells is very challenging and only mastered by a handful of laboratories world-wide. However, the quest for materials with relaxed requirements on the quality and better suitability for application in devices is opposed by the fundamental difficulty to realize a very ordered phase such as the BEC in disordered materials.

Very recently, our group at IBM Research - Zurich used an amorphous $35 \mathrm{~nm}$-thin polymer film embedded in an optical microcavity to achieve polariton BEC [5] (see Intro Illustration). The special luminescent polymer, which was developed about 20 years ago at the Max-Planck Institute for Polymer Research in Mainz for organic light emitting diodes (OLED), features a rigid backbone to reduce intrinsic disorder and enhance its optical properties.

\section{Can something as ordered as a Bose-Einstein condensate be created in something as disordered as a polymer fillm?}

By exciting the polymer film with picosecond laser pulses at a wavelength of $400 \mathrm{~nm}$, the transition from a normal phase to BEC shows up in the polariton dispersion (Fig. 1). The spectrum and angular distribution of the light coming from the microcavity allows to directly map out the dispersion relation and the occupation of the polariton states as a function of energy and momentum. At low pump intensity, the parabolic shape of the polariton dispersion is observed, where the intensity distribution reflects a Boltzmann distribution in energy and momentum - as expected for a thermalized ensemble of polaritons. When the intensity of the pump laser and therefore the polariton density is increased, suddenly a new peak appears which is much narrower in energy and momentum than the parabolic polariton dispersion. This is a key 
signature of the polariton BEC. Owing to the stimulated scattering mechanism responsible for the population of the BEC state, its intensity grows strongly super-linear with excitation power. It is slightly shifted to higher energy compared to the polariton parabola because of the repulsive Coulomb interaction in the dense ensemble of electron-hole pairs.

The most striking feature of a BEC is the emergence of long-range phase coherence where the wavefunctions of the condensing bosons are synchronized over macroscopic distances. This can be directly probed by sending the photons from the microcavity through a Michelson interferometer and recombining the light from the two interferometer arms on a camera. If the polaritons were coherent, they should interfere constructively and destructively and therefore form a fringe pattern. We observe such interference patterns over almost the whole pumped area which is several tens of micrometers in diameter (Fig. 2). Furthermore, certain characteristic patterns such as fork-like structures are indicative of the presence of vortex-like excitations in the condensate. As the total duration of this pulsed-driven BEC is currently on the order of a few picoseconds only, the observations are dominated by the "birth phase" of the condensate where vortices are expected to be quite ubiquitous [6]. Actually, this is a consequence of the so-called Kibble-Zurek mechanism that is a general feature of sudden phase transitions, and which was also at play in the formation of matter in the early universe.

Nearly two decades after the first atomic gas condensate just above absolute zero, we now have arrived at (non-equilibrium) BEC at room temperature in a plastic film. But apart from beautiful textbook-like quantum mechanical experiments, what is it good for in terms of applications? Most recently, atomic gas BECs have been used for "quantum simulations" of other physical systems, which are hardly experimentally

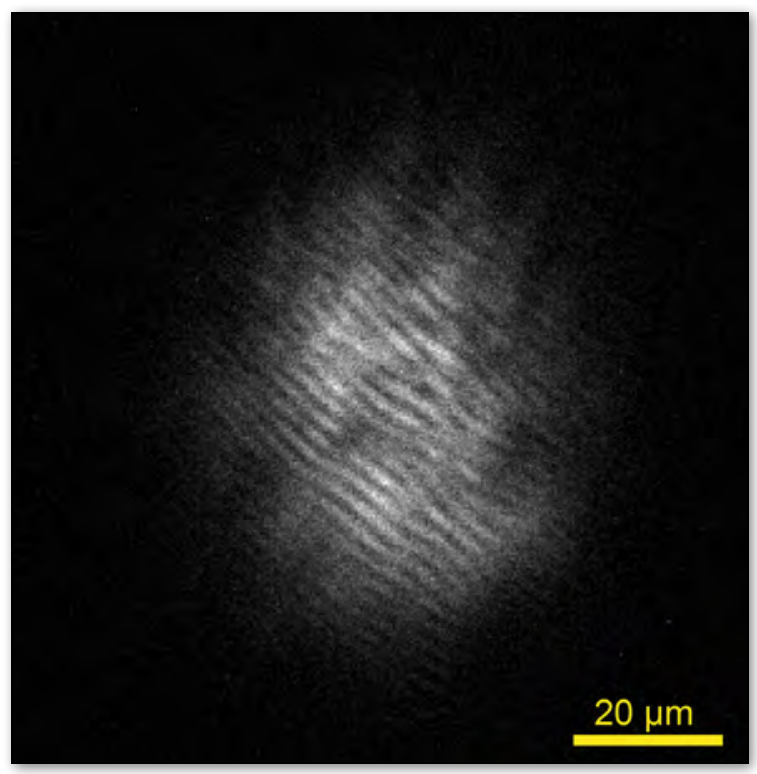

4 FIG. 2:

The interference pattern is a hallmark of the long spatial coherence length of the BEC. Non-parallel fringes are a consequence of excitations in the condensate. accessible or even not theoretically tractable [7]. For the solid-state polariton BEC, opto-electronic applications come within reach. Here the promise is to use the coherent laser-like light from the polariton BEC as micro-laser that does not require the energy-intensive creation of population inversion in the gain medium like in conventional lasers. The first electrically pumped polariton laser based on semiconductor quantum wells has just been demonstrated at a few degrees above absolute zero [8]. With the polymer-based BEC, however, electrical driving will be much more difficult, given the low charge mobility in the material, and will certainly require another quantum leap. Nevertheless, even optically driven polariton BECs are expected to provide deeper insight into fascinating quantum effects, especially simulating non-equilibrium phenomena on very fast timescales. And this has just become radically easier than ever before, something that Bose and Einstein probably would not have dreamed of almost 90 years ago.

\section{BOX: EXCITON-POLARITONS}

The optical activity of semiconductors, organics and most other materials originates from electron-hole pairs that can be formed by absorption of light or electrical excitation and can emit light by recombination. In the simplest approximation, this can be described as a two-level system with ground state (no exciton) and excited state (exciton has been formed). Placing such a material into a resonator that is tuned to the transition energy between the two states gives rise to new eigenstates of the coupled system, given that the interaction rate $\Omega$ between the cavity photon and the exciton is larger than the spontaneous emission rate $\gamma$ and the leakage rate $\mathrm{k}$ from the cavity. The resulting quasi-particles composed of half-exciton and half-photon are called exciton-polaritons and inherit the bosonic character from their constituents.

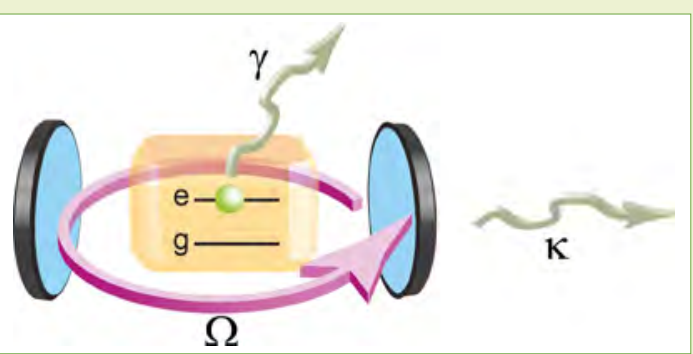




\section{About the authors}

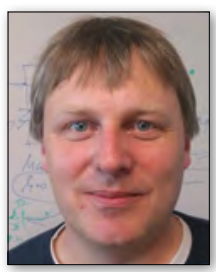

Thilo Stöferle received his PhD from ETH Zurich in 2005 for the investigation of BoseEinstein condensates and degenerate Fermi gases in optical lattices. In 2006 he joined IBM Research - Zurich where he is working on highly optical communication.

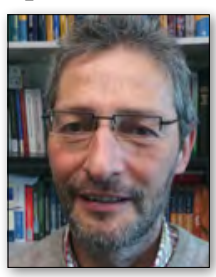

Rainer F. Mahrt received his $\mathrm{PhD}$ in 1991 and his habilitation in 1997 in Physical Chemistry from Marburg University. He was appointed a group-leader position at the Max-Planck Institute in 1999. In 2001 he joined IBM Research - Zurich. Current research interests are optics of organic/ inorganic hybrid systems in photonic band gap structures.

\section{References}

[1] M.H. Anderson, J.R. Ensher, M.R. Matthews, C.E. Wieman and E.A. Cornell, Science 269, 198 (1995)

[2] K.B. Davis, M.-O. Mewes, M.R. Andrews, N.J. van Druten, D.S. Durfee, D.M. Kurn and W. Ketterle, Phys. Rev. Lett. 75, 3969 (1995)

[3] A. Imamoğlu, R.J. Ram, S. Pau and Y. Yamamoto, Phys. Rev. A 53,4250 (1996)

[4] J. Kasprzak et al., Nature 443, 409 (2006)

[5] J.D. Plumhof, T. Stöferle, L. Mai, U. Scherf and R.F. Mahrt, Nature Materials 13, 247 (2014)

[6] C.N. Weiler, T.W. Neely, D.R. Scherer, A.S. Bradley, M.J. Davis and B.P. Anderson, Nature 455, 948 (2008)

[7] I. Bloch, J. Dalibard and W. Zwerger, Rev. Mod. Phys. 80, 885 (2008)

[8] C. Schneider et al., Nature 497, 348 (2013) 